\title{
Quadratic Stability Conditions of Linear Systems with Frobenius Norm-Bounded Diagonal Perturbations ${ }^{\dagger}$
}

\author{
Yoshiro Hamada*, Seiichi SHIN** and Noboru SEBE***
}

\begin{abstract}
This paper is concerned with quadratic stability of linear systems. The systems considered here have diagonally structured and Frobenius norm-bounded perturbations. An LMI-based quadratic stability condition for the systems is derived and a design method for the state feedback quadratic stabilizer is developed.
\end{abstract}

Key Words: quadratic stability, frobenius norm, diagonal perturbation, linear matrix inequality (LMI)

\section{Introduction}

Many researches have been made on quadratic stability of uncertain systems ${ }^{1) \sim 3)}$. While most of them deal with systems whose uncertainties are bounded by the twonorm or the maximum singular value, few papers investigate quadratic stability of systems with uncertainties bounded by another norm. The Frobenius norm is one of the important matrix norms and often appears in control theory ${ }^{4)}, 5$ ), which represents the average magnitude of all elements in the matrix.

J. H. Lee et. al. studied quadratic stability of linear systems with Frobenius norm-bounded uncertainties and derived its necessary and sufficient condition ${ }^{6)}$. They also treated linear systems with Frobenius norm-bounded $d i$ agonal uncertainties and derived a sufficient condition for quadratic stability ${ }^{7)}$. The condition is, however, not easily tractable because it consists of an infinite number of matrix inequalities.

In this paper we derive a new quadratic stability condition of linear systems whose uncertainties are diagonally structured and bounded by the Frobenius norm. The condition consists of just one matrix inequality, and a linear matrix inequality (LMI) based condition is also shown, which is available for analysis and synthesis. A quadratic stabilization method is proposed from the condition and the state feedback quadratic stabilizer can be easily designed using the method.

\section{Preliminaries}

We deal with the following uncertain system. Assume

$\dagger$ Presented in the 36th SICE Annual Conference $(1997 \cdot 7)$

* National Aerospace Laboratory, Jindaiji-Higashi 7-44-1, Chofu

** The University of Tokyo, Yayoi 2-11-16, Bunkyo-ku, Tokyo

*** Kyushu Institute of Technology, Kawazu 680-4, lizuka (Received December 6, 1999)

(Revised April 28, 2000) that the perturbation $\Delta$ is diagonally -structured and Frobenius norm-bounded:

$$
\begin{aligned}
& \dot{x}(t)=(A+B \Delta C) x(t), \quad\|\Delta\|_{F} \leq 1, \\
& \text { where } \quad x \in \Re^{n}, \Delta \in \Re^{r \times r}, \Delta=\left[\begin{array}{ccc}
\delta_{1} & & 0 \\
& \ddots & \\
0 & & \delta_{r}
\end{array}\right] .
\end{aligned}
$$

The Frobenius norm of the matrix is denoted by $\|\cdot\|_{F}$.

In 7), J. H. Lee et. al. derived sufficient condition for quadratic stability of uncertain systems. Originally the uncertainties in 7) are block diagonal and the quadratic sum of the block's maximum singular values is bounded. Applied to the system (1), the condition is reduced to the following lemma.

Lemma 1. An uncertain system (1) is quadratic stable if there exist a matrix $P>0$ and constants $0<\mu_{i}$ 's that satisfy the inequality

$$
\begin{gathered}
A^{T} P+P A+P B \mathcal{M}^{\frac{1}{2}} \Lambda^{2} \mathcal{M}^{\frac{1}{2}} B^{T} P \\
+C^{T} \mathcal{M}^{-\frac{1}{2}} \mathcal{M}^{-\frac{1}{2}} C<0 \\
\text { where } \Lambda=\left[\begin{array}{ccc}
\alpha_{1} & & 0 \\
& \ddots & \\
0 & & \alpha_{r}
\end{array}\right], \mathcal{M}=\left[\begin{array}{ccc}
\mu_{1} & & 0 \\
0 & & \mu_{r}
\end{array}\right],
\end{gathered}
$$

for all $\alpha_{i}$ 's such that $\sum_{i=1}^{r} \alpha_{i}^{2}=1$ and $\alpha_{i}>0(i=$ $1,2, \ldots, r)$.

The condition results in the infinite set of matrix inequalities, because Lemma 1 requires the system to satisfy the inequality (2) for all real $\alpha_{i}$ 's. Thus the condition cannot be solved in practice.

\section{Main Results}

In this section we develop a sufficient quadratic stability condition represented as a single inequality, which can be reduced to an LMI. To derive the condition, the region of perturbation $\left(\|\Delta\|_{F} \leq 1\right)$ is approximated to a larger region using a certain class of matrices ${ }^{8)}$.

Lemma 2. An uncertain system (1) is quadratic stable if there exist positive definite matrices $P$ and $Q_{a}=$ $\left\{q_{i j}\right\} \in \Re^{r \times r}$ that satisfy the inequality

$$
A^{T} P+P A+P B \tilde{Q}_{a} \tilde{Q}_{a}^{T} B^{T} P+C^{T} Q_{a}^{-T} Q_{a}^{-1} C<0,(3)
$$


where $\tilde{Q}_{a}$ is the diagonal matrix obtained from $Q_{a}$ :

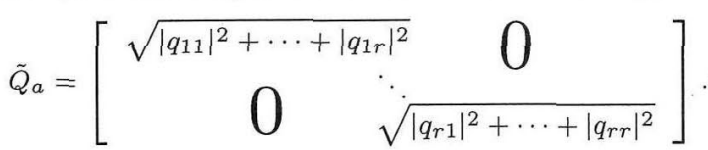

proof. The inequality (3) can be rewritten as follows:

$$
\begin{aligned}
A^{T} P+P A+ & P B \tilde{Q}_{a} \tilde{Q}_{a}^{T} B^{T} P \\
& +C^{T} \tilde{Q}_{a}^{-T} Q^{-T} Q^{-1} \tilde{Q}_{a}^{-1} C<0,
\end{aligned}
$$

where $Q=\tilde{Q}_{a}^{-1} Q_{a}$. The next inequalities are derived:

$$
\begin{aligned}
A^{T} P+ & P A+P B \tilde{Q}_{a} \tilde{Q}_{a}^{T} B^{T} P \\
& +C^{T} \tilde{Q}_{a}^{-T} Q^{-T} Q^{-1} \tilde{Q}_{a}^{-1} C \\
\geq & A^{T} P+P A+P B \tilde{Q}_{a} \Delta Q Q^{T} \Delta^{T} \tilde{Q}_{a}^{T} B^{T} P \\
& +C^{T} \tilde{Q}_{a}^{-T} Q^{-T} Q^{-1} \tilde{Q}_{a}^{-1} C \\
= & A^{T} P+P A+\left(P B \tilde{Q}_{a} \Delta Q-C^{T} \tilde{Q}_{a}^{-T} Q^{-T}\right) \\
& \times\left(P B \tilde{Q}_{a} \Delta Q-C^{T} \tilde{Q}_{a}^{-T} Q^{-T}\right)^{T} \\
& +P B \tilde{Q}_{a} \Delta \tilde{Q}_{a}^{-1} C+C^{T} \tilde{Q}_{a}^{-T} \Delta^{T} \tilde{Q}_{a}^{T} B^{T} P \\
\geq & A^{T} P+P A+P B \tilde{Q}_{a} \Delta \tilde{Q}_{a}^{-1} C \\
& +C^{T} \tilde{Q}_{a}^{-T} \Delta^{T} \tilde{Q}_{a}^{T} B^{T} P \\
= & A^{T} P+P A+P B \Delta C+C^{T} \Delta^{T} B^{T} P .
\end{aligned}
$$

Note that the first inequality is attained by

$$
\|\Delta Q\| \leq\|\Delta\|_{F} \leq 1
$$

where $\|\cdot\|$ denotes the maximum singular value. This is directly led by the diagonal structure of $\Delta$ and the fact that the Euclidean norm of each low vector in $Q$ equals to one. Then the derivative of the Lyapunov function $V(x)=x^{T} P x$ is bounded as

$$
\begin{aligned}
\dot{V}(x)= & \dot{x}^{T} P x+x^{T} P \dot{x} \\
= & x^{T}\left\{A^{T} P+P A+P B \Delta C+C^{T} \Delta^{T} B^{T} P\right\} x \\
\leq & x^{T}\left\{A^{T} P+P A+P B \tilde{Q}_{a} \tilde{Q}_{a}^{T} B^{T} P\right. \\
& \left.\quad+C^{T} \tilde{Q}_{a}^{-T} Q^{-T} Q^{-1} \tilde{Q}_{a}^{-1} C\right\} x . \quad
\end{aligned}
$$

Therefore, $\dot{V}(x)<0$. Hence the system is quadratic stable, if the condition (3) holds.

Note. The inequality (6) is equivalent to

$$
\left\|\tilde{Q}_{a}^{-1} \Delta Q_{a}\right\| \leq 1,
$$

since $\Delta$ and $\tilde{Q}_{a}^{-1}$ commute. The matrices $Q_{a}$ and $\tilde{Q}_{a}$ can be regarded as the scaling matrices for $\Delta$. Hence, using the scaled small gain theorem, the condition (3) can be also derived. See 8) for more detailed discussion from the viewpoint of the scaled small gain theorem.

The inequality (3) still cannot be easily solved because the relationship between $Q_{a}$ and $\tilde{Q}_{a}$ is not tractable. The problem is overcome by reformulating the condition as an LMI using the Schur complement. Since LMIs can be solved numerically, the following main theorem is useful

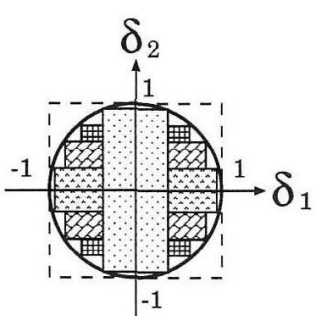

(a)

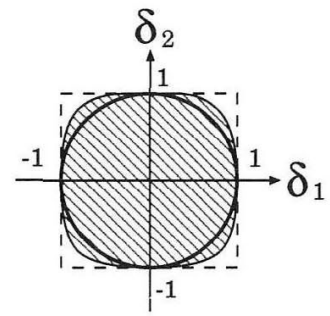

(b)
Fig. 1 The region $\|\Delta\|_{F} \leq 1$ is covered, (a): by the infinite set of polytopic regions, and (b): by just one region

for analysis of quadratic stability of the uncertain system considered here.

Theorem 1. An uncertain system (1) is quadratic stable if there exist positive definite matrices $X$ and $R$ that satisfy the LMI

$$
\left[\begin{array}{cc}
A X+X A^{T}+B \mathcal{D}(R) B^{T} & X C^{T} \\
C X & -R
\end{array}\right]<0,
$$

where $\mathcal{D}(\cdot)$ denotes the function that reduces all the nondiagonal elements of $(\cdot)$ to zero.

proof. Multiplication of $P^{-1}$ from the left and right hands of (3) leads us to

$$
X A^{T}+A X+B \tilde{Q}_{a} \tilde{Q}_{a}^{T} B^{T}+X C^{T} Q_{a}^{-T} Q_{a}^{-1} C X<0,
$$

where $X=P^{-1}>0$. The direct application of the Schur complement to this inequality results in

$$
\left[\begin{array}{cc}
A X+X A^{T}+B \tilde{Q}_{a} \tilde{Q}_{a}^{T} B^{T} & X C^{T} \\
C X & -Q_{a} Q_{a}^{T}
\end{array}\right]<0 .
$$

Since $\tilde{Q}_{a} \tilde{Q}_{a}^{T}=\mathcal{D}\left(Q_{a} Q_{a}^{T}\right)$ from the equation (4), the LMI condition (8) is obtained by letting $R=Q_{a} Q_{a}^{T}>0$.

Now we compare the condition in Theorem 1 derived here with that in Lemma 1 . It should be noted that, fixing the $\alpha_{i}$ 's, the condition (2) is equivalent to a sufficient condition for quadratic stability against diagonal polytopic perturbation $\Delta$ such that $\left|\delta_{i}\right| \leq \alpha_{i}$. Fig. 1 shows the region of $\Delta$ in the case of $r=2$ (the imaginary axes are ignored for simplicity). Each patterned rectangular region in Fig. 1(a) corresponds to one polytopic perturbation $\left|\delta_{i}\right| \leq \alpha_{i}$ for fixed $\alpha_{i}$ 's, and the perturbation $\|\Delta\|_{F} \leq 1$ is depicted as the region within the unit circle. As the figure indicates, the circular region $\|\Delta\|_{F} \leq 1$ can be covered by the infinite set of polytopic regions:

$$
\begin{aligned}
& \bigcup_{\boldsymbol{\alpha} \in \boldsymbol{U}}\left\{\Delta=\left[\begin{array}{cc}
\delta_{1} & 0 \\
0 & \delta_{2}
\end{array}\right]|| \delta_{1}\left|\leq \alpha_{1},\right| \delta_{2} \mid \leq \alpha_{2}\right\}, \\
& \text { where } \boldsymbol{U}=\left\{\boldsymbol{\alpha} \mid \alpha_{1}^{2}+\alpha_{2}^{2}=1\right\}, \quad \boldsymbol{\alpha}=\left[\begin{array}{ll}
\alpha_{1} & \alpha_{2}
\end{array}\right]^{T} .
\end{aligned}
$$

This is why the condition in Lemma 1 is composed of the infinite set of inequalities. On the other hand, the 
condition (3) is equivalent to a quadratic stability condition of the system with the diagonal perturbation $\Delta$ such that $\|\Delta Q\| \leq 1$, which is depicted as the shaded region in Fig. 1(b). The circular region $\|\Delta\|_{F} \leq 1$ is covered by one region $\|\Delta Q\| \leq 1$. Hence the condition in Theorem 1 is composed of just one inequality.

Note. A more tractable condition than (2) in Theorem 1 was also derived in 7 ) (Corollary 3) by letting $\Lambda=I_{r}$, where $I_{r}$ is the $r \times r$ identical matrix. This is just a quadratic stability condition against diagonal perturbation $\|\Delta\| \leq 1$. The perturbation is depicted as the region within the square in Fig. 1 (dotted line). From the figure, it can be seen that the condition is more conservative than that in Theorem 1.

We will also mention a design method for the quadratic stabilizer. Consider the following uncertain system:

$$
\begin{aligned}
& \dot{x}(t)=\left(A+D \Delta E_{a}\right) x(t)+\left(B+D \Delta E_{b}\right) u(t), \\
& \qquad\|\Delta\|_{F} \leq 1, \\
& \text { where } \quad x \in \Re^{n}, \Delta \in \Re^{r \times r}, \Delta=\left[\begin{array}{ccc}
\delta_{1} & & 0 \\
& \ddots & \\
0 & & \delta_{r}
\end{array}\right] .
\end{aligned}
$$

The following corollary gives a constant gain state feedback controller

$$
u(t)=-K x(t)
$$

that stabilizes the system.

Corollary 1. An uncertain system (9) is quadratic stabilizable via a state feedback control (10), if there exist matrices $X>0, R>0$ and $M$ that satisfy the LMI:

$$
\left[\begin{array}{cc}
\grave{z}+D \mathcal{D}(R) D^{T} & X E_{a}^{T}-M^{T} E_{b}^{T} \\
E_{a} X-E_{b} M & -R
\end{array}\right]<0,
$$

where $=A X+X A^{T}-B M-M^{T} B^{T}$.

Then a constant feedback gain can be obtained as $K=$ $M X^{-1}$.

proof. This is a straightforward application of Theorem 1.

\section{A Simple Example}

Let us consider the linear system with diagonal uncertainties described as

$$
\begin{aligned}
& \dot{x}(t)=\left(\left[\begin{array}{rr}
-2 & 1 \\
3 & -4
\end{array}\right]+\left[\begin{array}{cc}
\delta_{1} & 0 \\
0 & \delta_{2}
\end{array}\right]\right) x(t), \\
& \left\|\left[\begin{array}{cc}
\delta_{1} & 0 \\
0 & \delta_{2}
\end{array}\right]\right\|_{F}=\sqrt{\left|\delta_{1}\right|^{2}+\left|\delta_{2}\right|^{2}} \leq r .
\end{aligned}
$$

Using the condition in 6), where the structure of the perturbation is not considered, the bound is calculated as $r=0.9251$. And by the Corollary 3 in 7 ), the bound is obtained as $r=1.000$. Our condition, however, gives the larger bound $r=1.222$. This example shows that less conservative bound can be obtained using our quadratic stability condition.

\section{Conclusion}

We studied quadratic stability conditions for uncertain systems with Frobenius norm-bounded diagonal perturbations. The differences between Lee's condition in 7) and our condition were shown and our condition was proved less conservative. A design method for the quadratic stabilizer was also mentioned and a simple example of quadratic stability was provided. Tractable conditions for generally structured uncertain systems are left for further research.

\section{References}

1) I. R. Petersen: A Stabilization Algorithm for a Class of Uncertain Linear Systems, Systems \& Control Letters, 8, 351/357 (1987)

2) K. Zhou and P. P. Khargonekar: Robust Stabilization of Linear Systems with Norm-Bounded Time-Varying Uncertainty, Systems \& Control Letters, 10, 17/20 (1988)

3) P. P. Khargonekar, I. R. Petersen and K. Zhou: Robust Stabilization of Uncertain Linear Systems: Quadratic Stabilizability and $H^{\infty}$ Control Theory, IEEE Trans. on Automatic Control, 35-3, 356/361 (1990)

4) A. Dickman: On the Robustness of Multivariable Linear Feedback Systems in State-Space Representation, IEEE Trans. on Automatic Control, 32-5, 407/410 (1987)

5) S. Yamamoto, M. Kimura and R. Nagamune: An Identification Algorithm for a State-space Model Set Taking into Consideration Stabilizing State Feedback Control, Proc. of the 26th SICE Symposium on Control Theory, 277/280 (1997) (in Japanese)

6) J. H. Lee, W. H. Kwon and J. W. Lee: Quadratic Stability and Stabilization of Linear Systems with Frobenius Norm-Bounded Uncertainties, IEEE Trans. on Automatic Control, 41-3, 453/456 (1996)

7) J. H. Lee and W. H. Kwon: Quadratic Stability and Stabilization of Frobenius Norm Bounded Uncertain Systems, Proc. of the 1st Asian Control Conference, 499/502 (1994)

8) Y. Hamada, S. Shin and N. Sebe: Reliable Control and Frobenius Norm Optimization, Proc. of the 9th SICE Symposium on Decentralized Autonomous Systems, 169/172 (1997) (in Japanese) 